\title{
Global Synchronization Analysis For Complex Networks With Coupling Delay
}

\author{
Jinfang Zhang ${ }^{1}$, Yuanhua Qiao ${ }^{1}$, Jun Miao ${ }^{2}$, Lijuan Duan ${ }^{3}$ \\ ${ }^{1}$ College of Applied Sciences, Beijing University of Technology, Beijing 100124, China \\ ${ }^{2}$ Institute of Computing Technology Chinese Academy of Sciences, Beijing 100190, China. \\ ${ }^{3}$ College of Computer Science, Beijing University of Technology, Beijing 100124, China
}

\begin{abstract}
Global synchronization analysis for complex networks with coupling delay is investigated in this paper. The delay considered in this paper is constant. Sufficient conditions depending on the time delay are given based on Lyapunov functional, linear matrix inequality and Kronecker product technique. The unknown variables in the conditions are fewer and less conservative than those in the recent reference. Finally, two numerical examples are given to validate the effectiveness of the obtained results.

Index Terms - Complex networks, Global synchronization, Linear matrix inequality, Kronecker product.
\end{abstract}

\section{Introduction}

Dynamical recurrent neural networks are used extensively in classification of patterns, associative memories and optimization [1-3] etc. In these networks, there also exist more complicated dynamics, therefore, the theory of complex networks has become a focal research topic and attracted a great deal of attention in various fields. Recent years have witnessed an increasing interest in complex networks from the science and technology communities [4-6].

Recently, it is found that synchronization is one of the most important dynamical properties of complex networks and has been extensively investigated in different ways [7-13]. In [7], Lü and Chen considered a dynamical network and gave the conditions for local synchronization. Because of the network traffic congestions as well as the finite speed of signal transmission over the links, time delays occur commonly in complex networks. Therefore, Li, Zhou and Chen [8-9] improved the models with no delays, the case with constant coupling delays is considered, and they also analyzed the Synchronization problem [10-13]. Therefore, how to improve the system performance by removing the redundant variables and reducing computation still remain unsolved. Motivated by the mentioned discussions, we study the synchronization problems for general complex networks with time delay. Using different Lyapunov functionals given, the synchronization criteria derived turn out to be less conservative.

The remainder of this paper is organized as follows: in Section 2, the investigated systems are formulated and some lemmas and notations are given. In Section 3, the conditions for synchronization are derived. In Section 4, two numerical examples are presented to demonstrate the effectiveness and the advantage of the proposed method. Finally, conclusions are drawn in Section 5.

\section{II . Problem formulations}

Suppose the nodes are coupled with states $x_{i}(t)$, $i \in\{1, \cdots N\}$, then the dynamic neural networks (DNNs) of general form can be described by

$$
\begin{aligned}
\dot{x}_{i}(t)= & -C_{x}(t)+D f\left(x_{i}(t)\right)+B f\left(x_{i}(t-\tau)\right) \\
& +I(t)+c \sum_{j=l j \neq i}^{N} G_{i j}\left\{x_{j}(t-\tau)-x_{i}(t-\tau)\right] \\
& +c \sum_{j=j \neq i}^{N} G_{i j} A_{t}\left[x_{j}(t-\tau)-x_{i}(t)\right]
\end{aligned}
$$

in which $x_{i}(t)=\left[x_{i 1}(t), x_{i 2}(t), \cdots, x_{i_{n}}(t)\right]^{T} \in R^{n}$ is the state vector of the $i$ - th network at time $t$. The functions $f\left(x_{i}(t)\right)=\left[f_{1}\left(x_{i 1}(t)\right), f_{2}\left(x_{i 2}(t)\right), \cdots, f_{n}\left(x_{i_{n}}(t)\right)\right]^{T}$ is sufficiently smooth nonlinear vector fields, and $I(t)=\left[I_{1}(t), I_{2}(t), \cdots, I_{n}(t)\right]^{T} \in R^{n}$ is the external input vector, $C=\operatorname{diag}\left\{\left\{_{1}, c_{2}, \cdots, c_{n}\right\}>0, D=\left[d_{i j}\right]_{x \times n}, B=\left[b_{i j}\right]_{n \times n}\right.$, here $A=\left[a_{i j}\right]_{n \times n}$ and $A_{\tau}=\left[a_{i j}^{\prime}\right]_{n \times n}$ are inner-coupling matrix, $G=\left[G_{i j}\right]_{N \times N}$ represent the outer-coupling connections. The constant $c>0$ represents the coupling strength, $\tau$ represents the time constant delay.

For the network (1), the following assumptions are needed. Assumption (H1) The outer-coupling configuration matrices of the complex networks satisfy:

$$
\left\{\begin{array}{c}
G_{i j}=G_{j i} \geq 0, i \neq j, \\
G_{i i}=-\sum_{j=1, j \neq i}^{N} G_{i j}, i, j=1,2, \cdots, N .
\end{array}\right.
$$

Assumption (H2) For any constants $\sigma_{i}^{-}, \sigma_{i}^{+}$, the functions satisfy:

$$
\sigma_{i}^{-} \leq \frac{f_{i}\left(x_{1}\right)-f_{i}\left(x_{2}\right)}{x_{1}-x_{2}} \leq \sigma_{i}^{+}, \quad i=1,2, \cdots n
$$

we denote

$$
\Lambda_{1}=\operatorname{diag}\left(\sigma_{1}^{+} \sigma_{1}^{-}, \sigma_{2}^{+} \sigma_{2}^{-}, \cdots, \sigma_{n}^{+} \sigma_{n}^{-}\right),
$$




$$
\Lambda_{2}=\operatorname{diag}\left(\frac{\sigma_{1}^{+}+\sigma_{1}^{-}}{2}, \frac{\sigma_{2}^{+}+\sigma_{2}^{-}}{2}, \cdots, \frac{\sigma_{n}^{+}+\sigma_{n}^{-}}{2}\right)
$$

Based on Assumption (H1), system (3) can be rewritten as the following form:

$$
\begin{aligned}
\dot{x}_{i}(t)= & -C x_{i}(t)+D f\left(x_{i}(t)\right)+B f\left(x_{i}(t-\tau)\right) \\
& +I(t)+c \sum_{j=1}^{N} G_{i j} A x_{j}(t-\tau) \\
& +c \sum_{j=1}^{N} G_{i j} A_{\tau} x_{j}(t-\tau) \\
& -c G_{i i} A_{\tau}\left[x_{i}(t-\tau)-x_{i}(t)\right]
\end{aligned}
$$

The initial conditions of (2) are given by $x_{i}(s)=\phi_{i}(s) \quad . \quad s \in\left[t_{0}-\tau, t_{0}\right], \quad i=1,2, \cdots N \quad$.where $\phi_{i}(\cdot)=\left[\phi_{i 1}(\cdot), \phi_{i 2}(\cdot), \cdots \phi_{i n}(\cdot)\right]^{T} \in C\left(\left[t_{0}-\tau, t_{0}\right], R^{n}\right)$, and in order to simplify we set $G_{i i}=l$.

Remark 1: The constants $\sigma_{i}^{-}, \sigma_{i}^{+}$in assumption $\mathrm{H} 2$ are allowed to be any value. Then, most of the previous results in similar networks are just special cases of this assumption, which means that the activation functions are more general than that of other work.

Combining with the sign of Kronecker product, model (2) can be rewritten as:

$$
\begin{aligned}
\dot{x}(t)= & -\left(I_{N} \otimes C\right) x(t)+\left(I_{N} \otimes D\right) F(x(t))+\left(I_{N} \otimes B\right) \\
& F(x(t-\tau))+I^{\prime}(t)+c\left(G \otimes\left(A+A_{\tau}\right)\right) x(t-\tau) \\
& +c l\left(I_{N} \otimes A_{\tau}\right)[x(t-\tau)-x(t)]
\end{aligned}
$$

With $x(t)=\left[x_{1}^{T}(t), x_{2}^{T}(t), \cdots x_{N}^{T}(t)\right]^{T} \quad F(x(t))=\left[f^{T}\left(x_{1}(t)\right), f^{T}\left(x_{2}(t)\right)\right.$, $\left.\cdots, f^{T}\left(x_{N}(t)\right)\right]^{T}$ and $I^{\prime}(t)=\left[I^{T}(t), I^{T}(t), \cdots, I^{T}(t)\right]^{T}$.

Definition1 [12]: Dynamical networks (2) is said to be global asymptotically synchronization, if for any $\varepsilon$, any $\phi_{i}(s), \phi_{j}(s) \in C\left(\left[t_{0}-\tau, t_{0}\right], R^{n}\right) \quad(i, j=1,2, \cdots N)$. There exists $T>t_{0}$ such that $\left\|x_{i}(t)-x_{j}(t)\right\| \leq \varepsilon$, where $t>T, i, j=1,2, \cdots, N$.

and $\|\cdot\|$ denotes the Eulidean norm.

Lemma 1 Let $\otimes$ denote the notation of Kronecker product. Then, the following relationships hold
(1) $(\alpha A) \otimes B=A \otimes(\alpha B)$
(2) $(A+B) \otimes C=A \otimes C+B \otimes C$
(3) $(A \otimes B)(C \otimes D)=(A C) \otimes B D$

Lemma 2 [14] Let $e=(1,1, \cdots, 1)^{T}, E_{N}=e e^{T}, U=N I_{N}-E_{N}$, $P \in R^{n \times n}, x=\left(x_{1}^{T}, x_{2}^{T} \cdots x_{N}^{T}\right)^{T}, y=\left(y_{1}^{T}, y_{2}^{T} \cdots y_{N}^{T}\right)^{T}$ with $x_{k}, y_{k} \in R^{n},(k=1,2, \cdots N)$, then $x^{T}(U \otimes P) y=\sum_{1 \leq i<j \leq N}^{N}\left(x_{i}-x_{j}\right)^{T} P\left(y_{i}-y_{j}\right)$

Lemma 3 [15] For any constant matrix $X \in R^{n \times n}, X=X^{T} \geq 0$, a scalar functional $h:=h(t) \geq 0$, and a vector function $x:[-h, 0] \rightarrow R^{n}$, the following inequality hold $-h \int_{t-h}^{t} \dot{x}(s)^{T} X \dot{x}(s) d s \leq-[x(t)-x(t-h)]^{T} X[x(t)-x(t-h)]$

\section{III . Main Results}

In the section, we state and investigate the global asymptotically synchronization for the system (2).

Theorem 1: For given $\mathrm{c}, \tau>\mathbf{O}$, and supposing that Assumptions (H1)-(H2) hold, if there exists $P=P^{T}>0$, $Q=Q^{T}>0, W=W^{T}>0$, positive diagonal matrixes $H, V$ and matrix $T$ in appropriately dimension such that the following linear matrix inequalities (LMIS) hold for all $1 \leq i<j \leq N$.

$$
\begin{aligned}
& \Pi_{i j}=\left[\begin{array}{cc}
\alpha & -c N G_{i j} P\left(A+A_{\tau}\right)+c l P A_{\tau}+W \\
* & -Q-W-V \Lambda_{1} \\
* & * \\
* & * \\
* & *
\end{array}\right. \\
& \left.\begin{array}{ccc}
-C^{T} T^{T} & P D+H \Lambda_{2} & P B \\
\beta & 0 & V \Lambda_{2} \\
\tau^{2} W-T-T^{T} & T D & T B \\
* & -H & 0 \\
* & * & -V
\end{array}\right]<0
\end{aligned}
$$

Where $\alpha=-P C-C^{T} P+Q-c l P A_{\tau}-c l A_{\tau}^{T} P-W-H \Lambda_{1}$

$$
\beta=-c N G_{i j}\left(A+A_{\tau}\right)^{T} T^{T}+c l A_{\tau}^{T} T^{T} .
$$

Then system (3) is global asymptotical synchronization.

Proof: Choose a Lyapunov-Krasovskii functional candidate as:

$$
\begin{aligned}
V(x(t))= & x^{T}(t)(U \otimes P) x(t) \\
& +\int_{t-\tau}^{t} x^{T}(s)(U \otimes Q) x(s) d s \\
& +\int_{-\tau}^{0} \int_{t+\theta}^{t} \tau \dot{x}(s)^{T}(U \otimes W) \dot{x}(s) d s d \theta
\end{aligned}
$$

Now by directly computing $\dot{V}(x(t))$ along the trajectory of system (3), it can be deduced that 


$$
\begin{aligned}
\dot{V}(x(t))= & 2 x^{T}(t)(U \otimes P) \dot{x}(t)+x^{T}(t)(U \otimes Q) x(t) \\
& -x^{T}(t-\tau)(U \otimes Q) x(t-\tau) \\
& +\tau^{2} \dot{x}^{T}(t)(U \otimes W) \\
& \dot{x}(t)-\int_{t-\tau}^{t} \tau \dot{x}(s)^{T}(U \otimes W) \dot{x}(s) d s
\end{aligned}
$$

From Lemmal 3, we can obtain

$$
\begin{aligned}
& \dot{V}(x(t)) \leq 2 x^{T}(t)(U \otimes P) \dot{x}(t)+x^{T}(t)(U \otimes Q) x(t)- \\
& x^{T}(t-\tau)(U \otimes Q) x(t-\tau)+\tau^{2} \dot{x}^{T}(t)(U \otimes W) \dot{x}(t) \\
& -[x(t)-x(t-\tau)]^{T}(U \otimes W)[x(t)-x(t-\tau)]
\end{aligned}
$$

Noting the facts that $(U \otimes P) I(t)=0, U G=N G$, by Lemmal 1 and Lemmal 2 , we have

$$
\begin{aligned}
& \dot{V}(x(t)) \leq 2 \sum_{i=1}^{N-1} \sum_{j=i+1}^{N}\left(x_{i}(t)-x_{j}(t)\right)^{T}[(-P C+1 / 2 Q \\
& \left.-c l P A_{\tau}\right)\left(x_{i}(t)-x_{j}(t)\right)+P D\left(f\left(x_{i}(t)\right)-f\left(x_{j}(t)\right)\right) \\
& +P B\left(f\left(x_{i}(t-\tau)\right)-f\left(x_{j}(t-\tau)\right)\right)-c N G_{i j} P\left(A+A_{\tau}\right) \\
& \left.\left(x_{i}(t-\tau)-x_{j}(t-\tau)\right)+c l P A_{\tau}\left(x_{i}(t-\tau)-x_{j}(t-\tau)\right)\right] \\
& -\sum_{i=1}^{N-1} \sum_{j=i+1}^{N}\left(x_{i}(t-\tau)-x_{j}(t-\tau)\right)^{T} Q\left(x_{i}(t-\tau)-x_{j}(t-\tau)\right) \\
& +\tau^{2} \sum_{i=1}^{N-1} \sum_{j=i+1}^{N}\left(\dot{x}_{i}(t)-\dot{x}_{j}(t)\right)^{T} W\left(\dot{x}_{i}(t)-\dot{x}_{j}(t)\right) \\
& -\sum_{i=1}^{N-1} \sum_{j=i+1}^{N}\left[\left(x_{i}(t)-x_{j}(t)\right)^{T} W\left(x_{i}(t)-x_{j}(t)\right)-\right. \\
& 2\left(x_{i}(t)-x_{j}(t)\right)^{T} W\left(x_{i}(t-\tau)-x_{j}(t-\tau)\right)+\left(x_{i}(t-\tau)\right. \\
& \left.\left.-x_{j}(t-\tau)\right)^{T} W\left(x_{i}(t-\tau)-x_{j}(t-\tau)\right)\right]
\end{aligned}
$$

On the other hand, it is easy to see from the formulation of (3) that the following equation also holds for any matrices $T \in R^{n \times n}$ :

$$
\begin{aligned}
& 2 x^{T}(t)(U \otimes T)\left(-x(t)-\left(I_{N} \otimes C\right) x(t)+\left(I_{N} \otimes D\right) F(x(t))\right. \\
& +\left(I_{N} \otimes B\right) F(x(t-\tau))+I^{\prime}(t)+c\left(G \otimes\left(A+A_{\tau}\right)\right) \\
& x(t-\tau))+c l\left(I_{N} \otimes A_{\tau}\right)(x(t-\tau)-x(t))=0
\end{aligned}
$$

For convenience,

let $x_{i j}(t)=x_{i}(t)-x_{j}(t), f\left(x_{i j}(t-\tau)\right)=f\left(x_{i}(t-\tau)\right)-f\left(x_{j}(t-\tau)\right)$, $\dot{x}_{i j}(t)=\dot{x}_{i}(t)-\dot{x}_{j}(t)$, and $f\left(x_{i j}(t)\right)=f\left(x_{i}(t)\right)-f\left(x_{j}(t)\right)$.
For any $n \times n$ diagonal matrices $H>0, V>0$ and $\Lambda_{1}, \Lambda_{2}$ from Assumptions (H2), we have

$\sum\left\{-\left[x_{i j}^{T}(t) H \Lambda_{1} x_{i j}(t)-2 x_{i j}^{T}(t) H \Lambda_{2} f\left(x_{i j}(t)\right)+f^{T}\left(x_{i j}(t)\right)\right.\right.$

$\left.H f\left(x_{i j}(t)\right)\right]-\left[x_{i j}^{T}(t-\tau) V \Lambda_{1} x_{i j}(t-\tau)-2 x_{i j}^{T}(t-\tau) V \Lambda_{2}\right.$

$\left.\left.f\left(x_{i j}(t-\tau)\right)+f^{T}\left(x_{i j}(t-\tau)\right) V f\left(x_{i j}(t-\tau)\right)\right]\right\} \geq 0$

Adding up inequalities (8)-(10), we can get

$$
\dot{V}(t) \leq \sum_{i=1}^{N-1} \sum_{j=i+1}^{N}\left\{x_{i j}(t)^{T}(-P C+1 / 2 Q-c l P A) x_{i j}(t)+2 x_{i j}(t)^{T}\right.
$$

$\left.P D f\left(x_{i j}(t)\right)+2 x_{i j}(t)^{T} P B f x_{i j}(t-\tau)\right)+2 x_{i j}(t)^{T}\left(-c N G_{j j} P\left(A+A_{\varepsilon}\right)\right)$

$\left.x_{i j}(t-\tau)+2 x_{i j}(t)^{T}(c l P A) x_{i j}(t-\tau)\right\}-\sum_{i=1}^{N-1} \sum_{j=i+1}^{N}\left\{x_{i j}(t-\tau)^{T} Q_{i j}(t-\tau)\right.$

$+\sum_{i=1}^{N-1} \sum_{j=i+1}^{N} \tau^{2} \dot{x}_{i j}(t)^{T} W x_{i j}(t)-\sum_{i=1}^{N-1} \sum_{j=i+1}^{N}\left\{x_{i j}(t)^{T} W x_{i j}(t)-2 x_{i j}(t)^{T} W_{i j}(t-\tau)\right.$

$\left.+x_{i j}^{T}(t-\tau) W x_{i j}(t-\tau)\right\}-\sum_{i=1}^{N-1} \sum_{j=i+1}^{N}\left\{2 \dot{x}_{i j}^{T}(t) T \dot{x}_{i j}(t)+2 \dot{x}_{i j}^{T}(t) T C \gamma_{j}(t)\right.$

$\left.\left.-2 \dot{x}_{i j}^{T}(t) T D f x_{i j}(t)\right)-2 \dot{x}_{i j}^{T}(t) T B f x_{i j}(t-\tau)\right)+2 \dot{x}_{i j}{ }^{T}(t) c N G T\left(A+A_{i}\right)$

$\left.x_{i j}(t-\tau)-2 \dot{x}_{i j}^{T}(t)(c l T A) x_{i j}(t-\tau)\right\}-\sum_{i=1}^{N-1} \sum_{j=i+1}^{N}\left\{x_{i j}^{T}(t) H \Lambda x_{i j}(t)\right.$

$\left.-2 x_{i j}^{T}(t) H \Lambda_{2} f\left(x_{i j}(t)\right)+f\left(x_{i j}^{T}(t-\tau)\right) f\left(x_{i j}(t-\tau)\right)\right\}$

\section{$=\sum_{i=1}^{N-1} \sum_{j=i+1}^{N} \eta_{i j}^{T}(t) \Gamma_{i j} \eta_{i j}(t)$}

where $\Pi_{i j}$ is from (4), and

$\eta_{i j}^{T}(t)=\left[x_{i j}^{T}(t) \quad x_{i j}^{T}(t-\tau) \quad x_{i j}^{T}(t) \quad f\left(x_{i j}^{T}(t)\right) \quad f\left(x_{i j}^{T}(t-\tau)\right)\right] \quad$ Following theorm1, we have $V(t) \leq V(0)$, then we have $x_{i}(t)-x_{j}(t) \rightarrow 0$ $\forall 1 \leq i<j \leq N$. Therefore system (8) is global asymptotical synchronous. Then it completes the proof.

Remark 2: In order to obtain the better results, [13] used a inequality to instead of many free-weighting matrix. However, we use a new useful inequality to decrease the number of variables, and the result we obtained is better than them too.

\section{IV . Numerical Examples}

In the section, two examples are provided to illustrate the effectiveness of the conclusion.

Example1 Consider a lower-dimensional network model with 
5 nodes, where each node is a simple three-dimensional stable linear system. For simplify we assume that $C=\operatorname{diag}(1,2,3)$, $D=B=A_{\tau}=0, I(t)=0$, and the inner-coupling matrix is $A=\operatorname{diag}(1,1,1)$, the outer-coupling matrix is defined as:

$$
G=\left[\begin{array}{ccccc}
-2 & 1 & 0 & 0 & 1 \\
1 & -3 & 1 & 1 & 0 \\
0 & 1 & -2 & 1 & 0 \\
0 & 1 & 1 & -3 & 1 \\
1 & 0 & 0 & 1 & -2
\end{array}\right]
$$

We compare the admissible delay upper bounds for $\tau$ and the number of variables with those from other references, it is clearly from table 1 that the delay is a little larger and the number of variables are less than those from $[13,16]$.

Table 1 Admissible delay upper bound $\tau$ for different $c$

\begin{tabular}{|c|c|c|c|}
\hline Methods & Th.1 in[16] & Th.1 in[13] & Our result \\
\hline $\mathrm{c}=0.3$ & 1.345 & 1.632 & 1.666 \\
\hline $\mathrm{c}=0.4$ & 0.950 & 0.998 & 1.000 \\
\hline $\mathrm{c}=0.5$ & 0.731 & 0.732 & 0.898 \\
\hline $\begin{array}{l}\text { The number } \\
\text { of variables }\end{array}$ & More than 11 & 7 & 6 \\
\hline
\end{tabular}

Example2: We consider the following DNNs

$\dot{x}_{i}(t)=-C x_{i}(t)+D f\left(x_{i}(t)\right)+B f\left(x_{i}(t-\tau)\right)+I(t)$

Let us see the synchronized states of a chaotic system: Chua's circuit [17].

The dynamics of Chua's circuit is

$$
\left\{\begin{array}{l}
\frac{d x_{1}}{d t}=10\left(-x_{1}+x_{2}-f\left(x_{1}\right)\right) \\
\frac{d x_{2}}{d t}=x_{1}-x_{2}+x_{3} \\
\frac{d x_{3}}{d t}=-18 x_{2}
\end{array}\right.
$$

where $f\left(x_{1}\right)=b x_{1}+0.5(d-b)\left(\left|x_{1}+1\right|-\left|x_{1}-1\right|\right), d=-4 / 3, b=-3 / 4$.

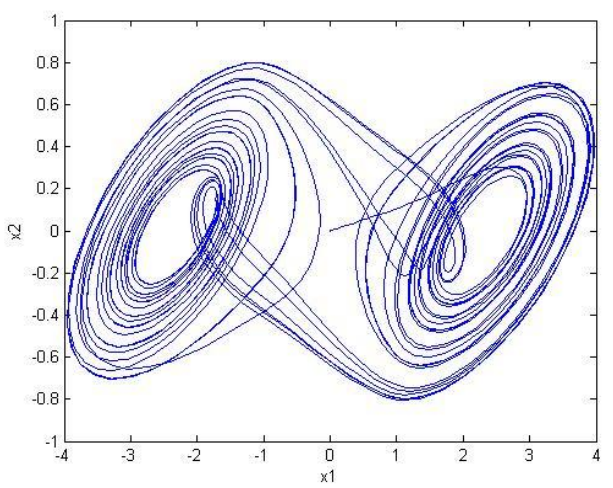

Fig.1 illustrates the chaotic trajectories of system (12).
Here we choose Chua's circuit (12) as the uncoupled system. The coupling time delay as $\tau=0.12$ and the coupling strength $c=1$, then we consider a dynamic networks consisting of three linearly coupled identical DNNs with couplings as

$$
\begin{aligned}
\hat{x}_{i}(t)= & -C x_{i}(t)+D f\left(x_{i}(t)\right)+B f\left(x_{i}(t-\tau)\right)+I(t) \\
& +c \sum_{j=1 j \neq i}^{N} G_{i j} A\left[x_{j}(t-\tau)-x_{i}(t-\tau)\right] \\
& +c \sum_{j=1 j \neq i}^{N} G_{i j} A_{\tau}\left[x_{j}(t-\tau)-x_{i}(t)\right]
\end{aligned}
$$

for $i=1,2,3$. Let $C=\left[\begin{array}{ccc}10 & -10 & 0 \\ -1 & 1 & -1 \\ 0 & 18 & 0\end{array}\right]$ and $D=\left[\begin{array}{ccc}-10 & 0 & 0 \\ 0 & 0 & 0 \\ 0 & 0 & 0\end{array}\right]$,

$G=\left[\begin{array}{ccc}-4 & 2 & 2 \\ 2 & -4 & 2 \\ 2 & 2 & -4\end{array}\right], A=\left[\begin{array}{lll}1 & 0 & 0 \\ 0 & 1 & 0 \\ 0 & 0 & 1\end{array}\right], A_{\tau}=\left[\begin{array}{ccc}0.5 & 0.1 & 0 \\ 0 & 0.5 & 0 \\ 0.3 & 0 & 0.5\end{array}\right]$,

$I(t)=B=0$, and $N=3, l=4$.

By calculating we have the activation functions $f\left(x_{i}\right)$ satisfy Assumption H3. If the system reaches synchronization, we have the following synchronized state equation:

$$
\dot{s}(t)=-C s(t)+D f(s(t))+B f(s(t-\tau))+l A_{\tau}(s(t-\tau)-s(t)) .
$$

By calculating other variables in theorem 1 Using Matlab Toolbox, we realize the system synchronization and the total error is given as $\operatorname{error}(t)=\sum_{i=1}^{3} \sqrt{\left[x_{1 i}-x_{2 i}\right]^{2}+\left[x_{2 i}-x_{3 i}\right]^{2}}$ Fig.2 shows the synchronized state of system (13) and the synchronous error with the initial value randomly chosen from $[0,1]$.

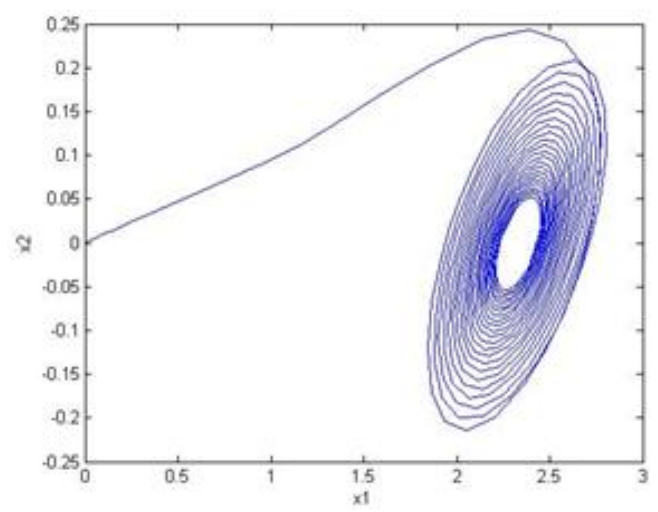




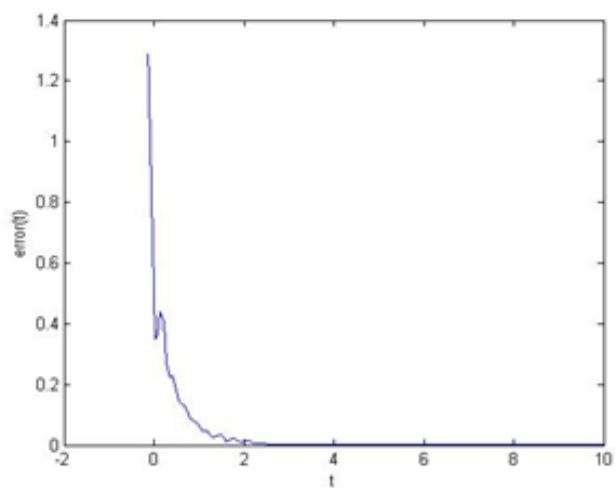

Fig.2 the synchronized state of system (13) and the synchronous error

\section{Conclusions}

In this paper, global synchronization problem is investigated for a general complex networks with coupling delays. A novel theorem is obtained about synchronization of the coupled systems by employing Lyapunov-Krasovskii functional. The condition obtained in this paper is expressed in the form of linear matrix inequalities, which has less variables and easy to be computed and checked by resorting to Matlab LMI Toolbox. The proposed network model may shed some new lights on the synchronization with one delay coupling. Furthermore, there are abundant dynamical behaviors in arrays of coupled systems with different coupling schemes and it deserves to be further studied in future. Finally, the example is utilized to illustrate the usefulness of the derived methods by simulation results.

\section{References}

[1] Wang J, Xu Z (2010) New study on neural networks: the essential order of approximation. Neural Netw 23:618-624.

[2] Wang G, Cao J, Wang L, Franklin J (2009) Global dissipativity of stochastic neural networks with time delay. J Franklin Inst 346:794-807.
[3] Zhang H, Wang Z, Liu D (2008) Global asymptotic stability of recurrent neural networks with multiple time-varying delays. IEEE Trans Neural Netw 19:855-873.

[4] Trestian I, Ranjan S, Kuzmanovic A, Nucci A (2010) Googling the internet: profiling internet endpoints via the World Wide Web. IEEE Trans Neural Netw 18:666-679.

[5] Wang YM, Xia J (2009) Unified framework for robust estimation of brain networks from fMRI using temporal and spatial correlation analyses. IEEE Trans Med Imaging 28:1296-1307.

[6] Yuan ZZ, Cai JP, and Lin ML (2010) Global synchronization in complex networks with adaptive coupling. Mathematical Problems in Engineering, Article ID 826721.

[7] Lü JH, Chen GR (2005) A time-varying complex dynamical network model and its controlled synchronization criteria. IEEE Transactions on Automatic Control, 50(6):841-846.

[8] Li CG, Chen GR (2004) Synchronization in general complex delayed dynamical networks with coupling delays. Physica A 343:263-278.

[9] Zhou J, Chen TP (2006) Synchronization in general complex delayed dynamical networks. IEEE Trans. Circuits Syst. I 53:733-744.

[10] Gao HJ, Lam J, Chen GR (2006) New criteria for synchronization stability of general complex dynamical networks with coupling delays .Phys. Lett. A 360:263-273.

[11] Li K, Guan SG, Gong XF (2008) Synchronization stability of general complex dynamical networks with time-varying delays. Phys. Lett. A 372: 713-727.

[12] He WL, C JD (2009) Global synchronization in arrays of coupled networks with one single time-varying delay coupling. Physics Letters A 373:2682-2694.

[13] Gong DW, Zhang HG (2011) New global synchronization analysis for complex networks with coupling delay based on a useful inequality. Neural Comput \& Applic.

[14] Wang Y, Wang ZD, Liang JL (2008) A delay fractioning approach to global synchronization of delayed complex networks with stochastic disturbances. Phys Letters A 372:6066-6073

[15] Shao HY (2009) New delay-dependent stability criteria for systems with interval delay. Automatica, vol. 45, no. 3, pp. 744-749.

[16] Li HJ, Yue D (2009) Synchronization stability of general complex dynamical networks with time-varying delays: A piecewise analysis method. J Comput Appl Math 232:149-158.

[17] L.0.Chua, (1992) The genesis of Chua's circuit. Arch. Elektron. Ubertragungstech., vol. 46, pp. 250-257. 